\title{
Biomimetic coating of apatite/collagen composite on Poly L-lactic Acid facilitates cell seeding
}

\author{
Yun Chen ${ }^{1}$, Arthur F.T. Mak ${ }^{1}$, Min Wang ${ }^{2}$, Jiashen $\mathrm{Li}^{1}$ \\ ${ }^{1}$ Jockey Club Rehabilitation Engineering Center, The Hong Kong Polytechnic University, Hong Hum, Hong Kong \\ ${ }^{2}$ Department of Mechanical Engineering, The University of Hong Kong, Pokfulam Road, Hong Kong
}

\begin{abstract}
Collagen and apatite were co-precipitated as a composite coating on poly L-lactic acid (PLLA) in an accelerated biomimetic process. The coating formed on PLLA films after 24 hours incubation was characterized. Saos-2 osteoblast-like cells were used to evaluate the cell seeding on this biomimetic composite coating. It was shown that cell seeding on PLLA films with the composite coating was greatly improved. PLLA coated with submicron collagen fibrils and submicron apatite paticulates can facilitate bone tissue engineering.
\end{abstract}

\section{INTRODUCTION}

Surface modification of biomaterials is a key issue in tissue engineering because material surface plays an important role in its interaction with host cells after biomaterials are implanted in-situ. Apatite coating has been widely used in bone tissue engineering for its high bioaffinity to cell and excellent osteoconductivity. A favorable strategy to make apatite coating would be to use the biomimetic process, since such process is carried out in mild condition. Besides the classical biomimetic process in SBF, supersaturated SBF (5 times SBF) [1] has been used to shorten the soaking period. We have also succeeded in forming bone-like apatite on degradable polymer films and scaffolds within 24 hours using this accelerated biomimetic process [2].

As another primary component of extracellular bone matrix, collagen has also been coated on various biomaterials, such as metals and polymers [3], to promote cell adhesion and bone integration.

In this work, to enhance the cell affinity of poly(Llactide) (PLLA), we combined collagen into the accelerated biomimetic process to form a complex composite coating of submicron apatite particulates and collagen fibers on PLLA films. The final microstructural and composition of the coating formed were investigated. The cell affinity of the PLLA film with composite coating was also evaluated and compared with PLLA film without coating and with apatite coating alone.

\section{MATERIALS AND METHODS}

Materials PLLA with an inherent viscosity of approximately $7.11 \mathrm{dl} / \mathrm{g}$ was purchased from PURAC
(Netherlands). Chloroform and all the analytical grade chemicals for making SBF were purchased from Acros (Belgium). Type-I Collagen was obtained from Tsinghua University in Beijing, China. The osteoblastlike cells, Saos- 2 cells, were supplied by ATCC (USA).

Fabrication of PLLA films PLLA films were fabricated by the solvent-evaporation method. Typically, PLLA granules were dissolved in chloroform. Then the solvent evaporated away. PLLA films with an even thickness $(\sim 0.1 \mathrm{~mm})$ were obtained.

Incubation solution The chemical compositions of $5 \mathrm{SBF}$ and 5SBFC were summarized in table $1 . \mathrm{pH}$ value was adjusted to 6.4 with $\mathrm{HCl}$ and Tris in both $5 \mathrm{SBF}$ and 5SBFC solution.

Table 1. Compositions and ion concentrations (mM) of 5SBF and

\begin{tabular}{|l|l|l|}
\hline \multicolumn{3}{|c|}{ 5SBFC. } \\
\hline $\mathrm{Na}^{+}$ & $5 \mathrm{SBF}$ & $5 \mathrm{SBFC}$ \\
\hline $\mathrm{K}^{+}$ & 710.0 & 710.0 \\
\hline $\mathrm{Ca}^{2+}$ & 25.0 & 25.0 \\
\hline $\mathrm{Mg}^{2+}$ & 12.7 & 12.7 \\
\hline $\mathrm{Cl}^{-}$ & 7.7 & 7.7 \\
\hline $\mathrm{HCO}_{3}{ }^{-}$ & 739.7 & 739.7 \\
\hline $\mathrm{HPO}_{4}{ }^{2-}$ & 21.0 & 21.0 \\
\hline $\mathrm{SO}_{4}{ }^{2-}$ & 5.0 & 5.0 \\
\hline malonic acid & 2.5 & 2.5 \\
\hline collagen & $/$ & $3 \mathrm{~g} / \mathrm{L}$ \\
\hline
\end{tabular}

Incubation of PLLA films Three square PLLA films $(10 \mathrm{~mm} \times 10 \mathrm{~mm} \times 0.1 \mathrm{~mm})$ were immersed in $50 \mathrm{~mL} 5 \mathrm{SBF}$ or $5 \mathrm{SBFC}$ in a plastic bottle in a shaker bath set at $60 \mathrm{rpm}$ with the temperature maintained at $37^{\circ} \mathrm{C}$. After 24 hours of incubation, the specimens were taken out of the bottles, washed carefully with distilled deionized water to remove the soluble inorganic ions, and then dried in air.

Chemical Characterizations The coating formed was viewed under a LEICA scanning electron microscope (Model Stereoscan 440) after being coated with gold. The elemental composition of surface was examined with energy dispersive X-ray spectrometer (EDX) without gold coating. Thin-film X-ray diffraction (XRD) spectra were obtained by a Philips X-ray 
diffractometer with a fixed incidence of $1^{\circ}$ in the range of $10-40^{\circ}$ using $0.06^{\circ}$ step and $1 \mathrm{~s} /$ step scan speed. The transmission spectra of the samples were measured with a Perkin-Elmer FTIR Spectrometer. A small amount of coating was scratched from the surface of substrates. It was then milled with potassium bromide $(\mathrm{KBr})$ and pressed into a transparent film for FTIR analysis.

Cell adhesion Assay The samples were placed into 24well polystyrene plates after being washed three times with PBS solution. Saos-2 cells were suspended in Dulbecco's modified eagle medium containing 5\% serum and $1 \%$ penicillin/streptomycin. 100ul (about $4 \times 10^{4}$ cells) of the cell suspensions were placed on the samples and cultured for $4 \mathrm{~h}$ before $1 \mathrm{ml}$ culture medium was added into each well. The plate was incubated at a temperature of $37^{\circ} \mathrm{C}$ in a $5 \% \mathrm{CO}_{2}$ atmosphere. After incubation, any non-adherent cells on the samples were removed by aspirating the medium and washing with PBS solution.

The samples, after having been cultured for 2 hours, were taken out of the culture plates and washed with PBS three times. Thereafter, the samples were fixed with $3 \%$ glutaraldehyde in PBS for 24 hours at $4^{\circ} \mathrm{C}$. After being thoroughly washed with PBS, the samples were dehydrated sequentially in $30,50,70,80,90,95,100 \%$ ethanol each for $2-10 \mathrm{~min}$. the fixed samples were freeze-dried, sputter-coated with gold, and examined under a LEICA scanning electron microscope (Model Stereoscan 440).

In the quantitative assay, the adherent cells after being cultured for 3 days were removed from the samples by trypsinization. The viable cells were counted using a hemocytometer. For each type of substrates, 5 samples were used to obtain a mean value and standard deviation for the number of adherent cells.

\section{RESULTS \& DISCUSSION}

EDX results shown in Fig. 1(a) indicated that calcium and phosphorus elements existed in both of the two types of coatings formed on PLLA films. In the XRD pattern (Fig.1(b)), compared with pure PLLA films (A), PLLA films after incubation in 5SBF (B) and in 5SBFC $(\mathrm{C})$ revealed the characteristic peaks of apatite. The FTIR spectra acquired from the coating formed in $5 \mathrm{SBF}$ and $5 \mathrm{SBFC}$ were displayed in Fig. 1(c). In the spectra of the coating formed by the accelerated biomimetic coprecipitation process in 5SBFC (B), besides the same functional groups, $\mathrm{PO}_{4}{ }^{3-}$, $\mathrm{CO}_{3}{ }^{2-}$, and $\mathrm{OH}^{-}$, as in $5 \mathrm{SBF}(\mathrm{A})$, amide peaks are also revealed. These analyses suggest that an apatite/collagen layer formed on the PLLA surface after immersion in 5SBFC for only 1 day.

The SEM micrograph in Fig. 2 showed the surface morphologies of the PLLA films after incubation in $5 \mathrm{SBF}$ and $5 \mathrm{SBFC}$ for 24 hours. The films immersed for 24 hours in 5SBF and 5SBFC [Fig. 2(a),(b)] exhibited the apatite coating and the apatite/collagen composite coating, respectively. In the SEM micrograph of PLLA films with apatite/collagen composite coating [Fig. 2(b)], some submicron apatite particles (100-200nm) were observed on the collagen fibers, as well as on the PLLA surface. The diameters of fibers were also submicron. In some places, deposited fibers interlapped randomly with each other. Natural bone is a composite in which apatite particulates are aligned on the collagen fibers at the $\mathrm{nm}$ to $\mu \mathrm{m}$ scale. This combined apatite/collagen coprecipitation in 5SBFC allowed the formation of a submicron biocomposite of collagen and apatite in composition and scale more similar to natural bone.

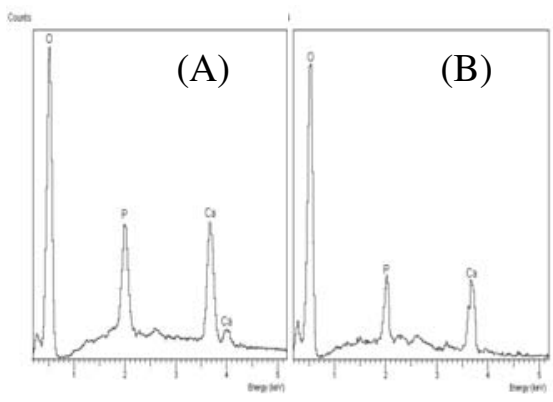

(a)

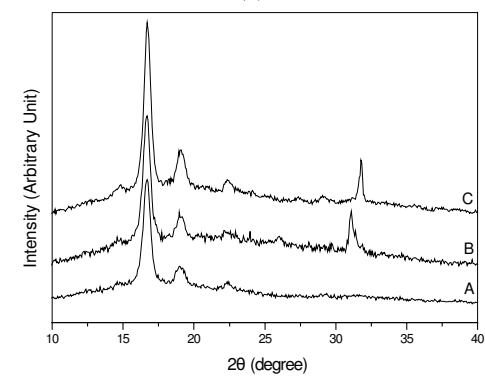

(b)

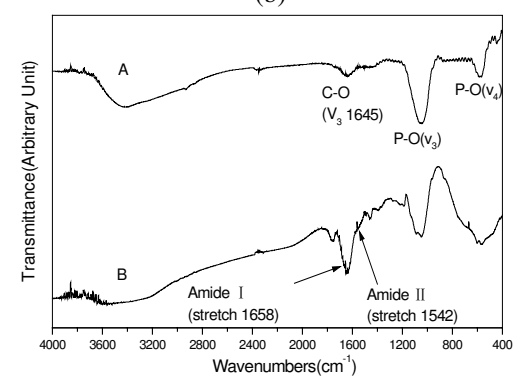

(c)

Fig 1. (a) EDX spectra of PLLA films incubated for 24 hours in (A) 5SBF (B) 5SBFC; (b) XRD patterns of PLLA films (A) without treatment (B) incubated in 5SBF for 24 hours $(C)$ incubated in 5 SBFC for 24 hours.; (c) FTIR spectrum of PLLA films incubated for 24 hours in (A) $5 \mathrm{SBF}$ (B) $5 \mathrm{SBFC}$.

Based on the mechanism of apatite formation on other substrates [4], the possible mechanism of apatite and collagen formation on the PLLA in 5SBFC is therefore suggested as follows. PLLA hydrolysis may generate acidic groups $-\mathrm{COOH}$ on the surface, which may facilitate collagen absorption. The groups of $\mathrm{COOH}$ may also further change to $-\mathrm{COO}^{-}$, providing a negatively charged surface to adsorb positive $\mathrm{Ca}^{2+}$ and 
induce apatite nucleation. The functional groups existing on the surface of collagen, such as $-\mathrm{COOH}$ and $-\mathrm{NH}_{2}$, may also become charged groups, such as $\mathrm{COO}^{-}$and $-\mathrm{NH}_{3}{ }^{+}$, which again could absorb positive $\mathrm{Ca}^{2+}$ and negative $\mathrm{HPO}_{4}{ }^{2-}$ to form apatite nuclei. Hence apatite particulates could form on the surface of collagen and PLLA.

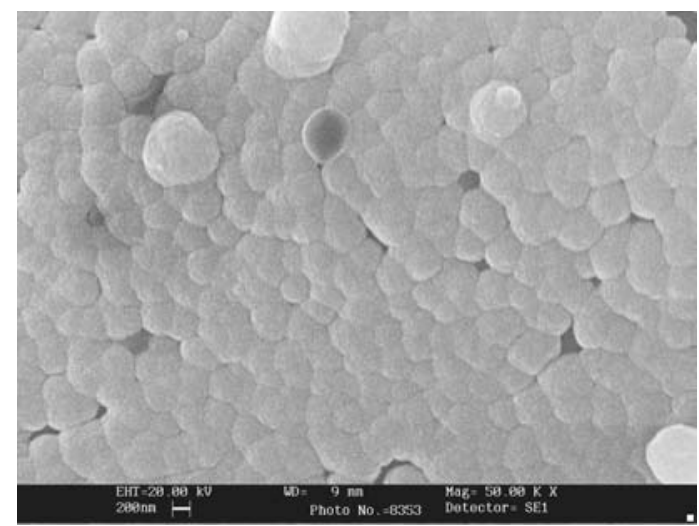

(a)

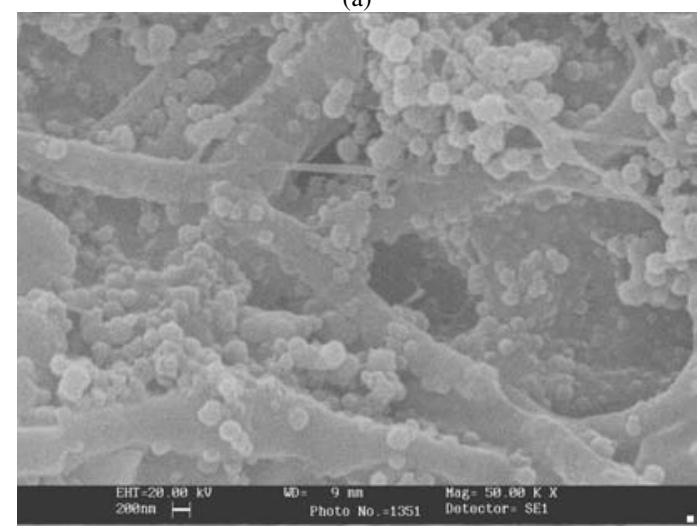

(b)

Fig 2. SEM micrographs (magnification 50,000x) of PLLA films (a) with apatite coating; (c) with apatite/collagen compoiste coating

It was confirmed from the SEM observations (Fig.3) that more cells were attached on the composite coating and they had stretched their pseudopodia well, whereas fewer cells was observed on the PLLA substrate and they exhibited a rounded shape. Cell number and morphology on apatite coating were between that on PLLA substrate and composite coating.

Fig. 4 showed the number of adherent cells on each samples after 3 days culture. It could be noted that the number of cells attached to the composite coating was significantly higher than that on the PLLA substrate and the apatite coating.

The present biomimetic composite coating is, therefore, found to be much more effective in improving the cell-adhesive properties. The reason of this superiority may be attributed to the similarity of such composite coating with nature bone in composite and scale.

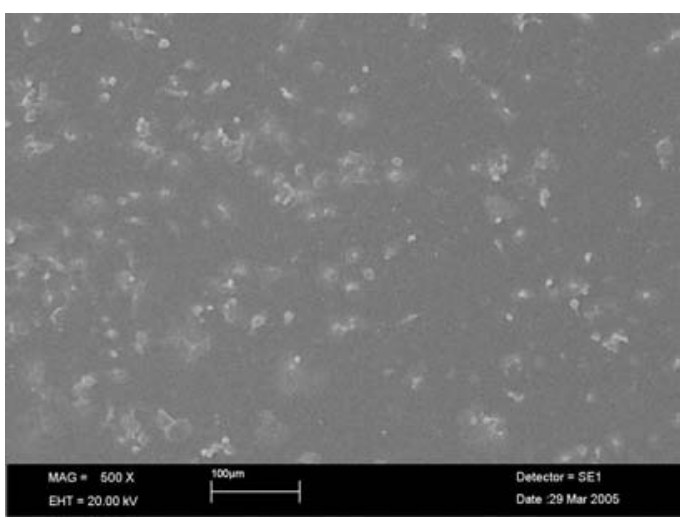

(a)

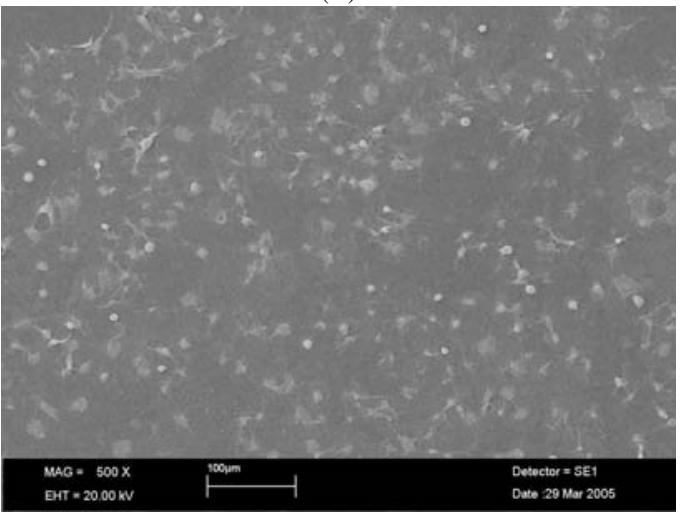

(b)

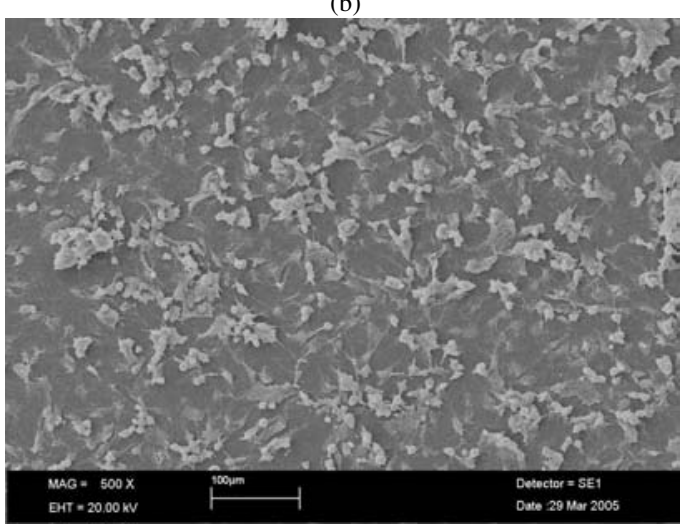

(c)

Fig. 3 SEM image of Saos- 2 cells after $2 \mathrm{~h}$ culture on PLLA film (a) without coating, (b) with apatite coating, (c) with apatite/collagen composite coating.

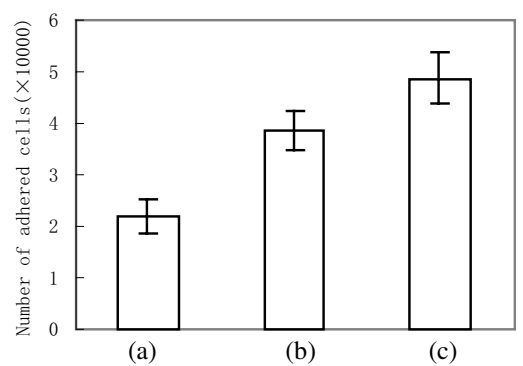

Fig. 4 Comparison of cell growth on PLLA films after 3 days culture (a) without coating, (b) with apatite coating, (c) with apatite/collagen composite coating 
Although only PLLA was used here as an example of substrates, other biomaterials, metal or polymer, dense or porous, may also be coated with this composite coating using this accelerated biomimetic coprecipitation process. Therefore, a variety of biomaterials with this biomimeitc composite coating for biomedical applications may be obtained.

\section{CONCLUSIONS}

Biomimetic composite coating of bone-like submicron apatite particles combined with collagen fibers was formed on PLLA films within 24 hours by an accelerated biomimetic coprecipitation process. This coating showed a composition more relevant to that of natural bone than apatite coating or collagen coating alone. The cell-adhesive properties of such biocomposite coating were superior to apatite coating and PLLA substrate. The PLLA coated with collagen submicron fibrils and apatite submicron paticulates are expected to be useful as a novel template for bone tissue engineering.

\section{REFERENCES}

[1] Barrere F, Blitterswijk CA, Groot K, Layrolle P. Influence of ionic strength and carbonate on the $\mathrm{Ca}-\mathrm{P}$ coating formation from SBF×5 solution. Biomaterials 2002;23(9):1921-1930.

[2] Chen Y, Mak AFT, Li J, Wang M, Shum AWT. Formation of apatite on poly ( $\alpha$-hydroxy acid) in an accelerated biomimetic process. J Biomed Mater Res. Part B. 2005;73B(1):68-76

[3] Suh H, Hwang YS, Lee JE, Han CD, Park JC. Behavior of osteoblasts on a type I atelocollagen grafted ozone oxidized poly L-lactic acid membrane. Biomaterials 2001;22:219-230.

[4] Liu X, Ding C, Chu PK. Mechanism of apatite formation on wollastonite coatings in simulated body fluids. Biomaterials 2004;25:1755-1761. 